Preprint of the:

Montanyà et al. (2015), X-rays and microwave RF power from high voltage laboratory sparks, Journal of Atmospheric and Solar-Terrestrial Physics, http://dx.doi.org/10.1016/j.jastp.2015.06.009

\title{
X-rays and microwave RF power from high voltage laboratory sparks
}

Joan Montanyà*, Ferran Fabró, Víctor March, Oscar van der Velde, Glòria Solà, David Romero, Oriol Argemí

Universitat Politècnica de Catalunya, Terrassa, Barcelona, Spain

(*corresponding author telephone: +34 937398 071; e-mail: montanya@ee.upc.edu)

\begin{abstract}
Lightning flashes involve high energy processes that still are not well understood. In the laboratory, high voltage pulses are used to produce long sparks in open air allowing the production of energetic radiation. In this paper X-rays emitted by long sparks in air are simultaneously measured with the RF power radiation at $2.4 \mathrm{GHz}$. The experiment showed that the measured RF power systematically peaks at the time of the X-rays generation (in the microsecond time scale). All of the triggered sparks present peaks of RF radiation before the breakdown of the gap. The RF peaks are related to the applied voltage to the gap. RF peaks are also detected in discharges without breakdown. Cases where X-rays are detected presented higher RF power. The results indicate that at some stage of the discharge, before the breakdown, electrons are very fast accelerated letting in some cases to produce X-rays. Microwave radiation and X-rays may come from the same process.
\end{abstract}




\section{Introduction}

Productions of X-rays from electrical discharges at atmospheric pressure have been studied for a long time (e.g. Stankevich and Kalinin, 1967). The recent discoveries of the Terrestrial Gamma ray Flashes (TGF) by Fishman et al. [1994] and the high-energy emissions produced by lightning [Moore et al., 2001] promoted the interest of the atmospheric electricity community in the high energy emissions produced by laboratory discharges at air [e.g. Dwyer et al., 2005; Nguyen et al., 2008; Rahman et al., 2008; March and Montanyà, 2010 and 2011; Kochkin et al.,2012]. One of the interests in high voltage experiments is to understand the mechanisms of the production of energetic radiation that occurs in lightning and may probably share similar properties than the intense TGF to space. Results from laboratory experiments showed the importance of the overvoltage attained in the inter-electrode gap in order to produce X-rays (e.g. Babich and Loiko, 2010 and related references therein). In that way, March and Montanyà [2010] showed how a fast voltage growth lets to higher probability of X-ray production and with higher energies. In addition, the asymmetry between streamer/leader polarities related to the energetic production was studied by March and Montanyà [2011].

Radio Frequency (RF) radiation from lightning at frequencies higher than $500 \mathrm{MHz}$ were first recorded by Takagi and Takeuti [1963] and later by Brook and Kitagawa [1964]. They found that most of the RF radiation was associated with stepped leaders, dart leaders and k-changes (recoil leaders). Later, Kosarev et al. [1970] suggested that the radiation at decimetric wave range is different from the dipole emission from the lightning currents. Rust et al. [1979], by measuring at $2.2 \mathrm{GHz}$, found bursts of radiation during the preliminary breakdown, in conjunction with the initial return stroke and during dart leaders 
of subsequent strokes. Recently Petersen and Beasley [2013a and 2013b] presented radiation at $1.57 \mathrm{GHz}$ from negative stepped leaders.

Bekefi and Brown [1961] studied bremsstrahlung radiation produced by accelerating electrons. When an electron makes a transition between two continuous states with initial and final energies of $m v_{\mathrm{i}}^{2} / 2$ and $m v_{\mathrm{f}}^{2} / 2$ respectively, it radiates and emits a quantum of $\hbar \omega$. Due to the transitions between continuous states the radiation forms a continuum. Later, Rai et al. [1972] based on the previous reference of Bekefi and Brown [1961], found that the bremsstrahlung process is a possible source of RF emissions in UHF and microwaves measured during some lightning processes. The authors demonstrated how the full ionized plasma in the return stroke does not radiate microwave power since its absorption coefficient lets to an optical thickness much greater than the unity. On the other hand, the partially ionized plasma in the stepped leader, dart leader and k-changes (recoil leader) does emit microwaves. In such cases the estimated optical thickness is lower than the unity due to the negligible absorption. Microwave radiation can be basically due to electron-atom encounters. Bondiou [1987] studied the V-UHF RF emission of laboratory discharges and proposed a model. The impulsive RF radiation was associated with the processes during the formation of the transient arc and could be similar to the produced by lightning. The called transient arc phase in the breakdown takes place between the streamer phase and the development of the conductive channel with duration between 5 to $10 \mathrm{~ns}$. Recently, Cooray and Cooray [2012] calculated the radiation of electron avalanches showing that can radiate in the microwave region.

In this paper, we present simultaneous measurements of X-rays and microwave RF power emissions at $2.4 \mathrm{GHz}$ from high voltage sparks. The simultaneous occurrence of the 
X-rays and the absolute RF peaks are presented and discussed. The emission of microwave $\mathrm{RF}$ is studied for negative and positive discharges with different gap voltage. A discussion of the obtained results and the possible mechanism is addressed.

\section{Experimental setup}

The geometries studied in this paper corresponds vertical rod-rod and rod-plate setups. In both cases, the lower electrode was grounded whereas the rod on top was connected to the output of a 1.2 MV Marx generator. The rod was round terminated with a diameter of $16 \mathrm{~mm}$ and made from stainless-steel. The gap varied from $60 \mathrm{~cm}$ to $1 \mathrm{~m}$. The adjusted waveform presented and average rise time of $0.55 \mu \mathrm{s}$. The instruments where placed within an EMI shielded cabined. The measurement cabinet was placed horizontally from the gap. The cabinet contained a scintillation detector $(\mathrm{NaI}(\mathrm{Tl})$ and $\mathrm{LaBr} 3)$ and a receiver tuned at 2.4 GHz with a pass-band of 5.5 MHz. The receiver was based on a logarithmic detector with a dynamic range from $-65 \mathrm{dBm}$ to $+10 \mathrm{dBm}$ with a SAW filter in order to tune it at the desired frequency. The receiver was calibrated by means of a RF signal generator. The antenna used was a microstrip antenna adjusted at the tuned frequency. The estimated gain of the antenna was $<10 \mathrm{dBi}$.

The results presented in this paper correspond to two sets of experiments. In the first set a rod-rod gap was setup with a gap distance of $68 \mathrm{~cm}$ and the instrumented cabinet separated $0.8 \mathrm{~m}$ from the gap. The object of this experiment was to investigate if there is any characteristic of the RF emissions related to the production of X-rays and. In the second set, the effect of the peak voltage on the RF emissions was studied for both polarities. In this last experiment the measurement cabinet was at $1.6 \mathrm{~m}$ from the gap. 


\section{Results of simultaneous detection of X-rays and RF pulses}

$\mathrm{X}$-rays and signals of RF power were measured from rod-rod sparks with negative polarity. As found in previous works (e.g. March and Montanyà, 2010), only discharges with negative polarity produced X-rays. Figure 1 displays two examples of the recorded Xrays and RF power. The figure shows how RF power peaks at the time (with the adjusted time scale) of the X-ray pulses. This observation was systematically repeated for more than 25 impulses in which X-rays were detected. During the entire discharge process the absolute RF maximum is coincident with the detection of X-rays. The dashed lines indicate the time of breakdown, note that the breakdown neither produce emission at the considered frequency and in X-rays.

In order to investigate any possible relation between the peak of the RF power and the $\mathrm{X}$-rays, figure 2 plots the measured energy versus the RF power for ten cases. The small sample in figure 2 does not show evidence of straight relation between the peak of the RF power and the energy of the X-ray pulses. All the cases where X-rays were detected presented RF power levels higher than $-20 \mathrm{dBm}$. However no clear relation between both magnitudes is found here.

\section{Results of the relation between peak voltage and RF power}

The second experiment was focused on the study of the relation between the peak of the RF power and the applied voltage. In this case more than 40 impulses are considered. Within these impulses in only one discharge X-rays were detected. The object here is to show the relation between the peak of the applied voltage into the gap and the measured RF power. From the graphs in figure 3 we can find how at low peak voltages, the RF power emissions are in general lower than at higher voltages. Moreover, discharges that produced 
breakdown tend to produce higher levels of RF power. The case marked as \#1 in Figure 3 corresponds to the singular case with detection of X-rays. Note that this case presents a singularity in the dataset for the negative polarity impulses. On the other hand, the other singular case labeled as \#2 corresponds to an intense RF power detection in a positive impulse. Despite the intense RF emission no X-rays were detected.

\section{Discussion}

The results shown in the previous figures suggest that the mechanism of the peaks of $\mathrm{RF}$ power at microwave frequencies is in common with the production of X-rays. As indicated by Rai et al. [1972] based on Bekefi's works, UHF emission from lightning would be produced by bremsstrahlung process. Under this hypothesis, lightning leaders would be much more efficient radiating at microwaves than return stroke channels. Here we found similar results with the discharges at the laboratory but with the extension to higher energies. The signals in figure 1 revealed how RF power peaks during the streamer (and possibly leader) phase whereas during the breakdown and the high current of the spark the RF signals do not show any effect. In addition to bremsstrahlung radiation, our measurements could be explained considering the radiation from electron avalanches as calculated by Cooray and Cooray [2012]. In the cases with X-ray generation an enhancement of the streamer activity at the time of the X-rays would explain the increase of radiation.

In the experiments here we did not find any straight relation between the energy of the X-rays and the RF peaks. We believe that some relation may exist since March and Montanya [2010] found that energy of the high energy radiation was related with voltage growth $\Delta \mathrm{V} / \Delta \mathrm{t}$ during the initial phase of the discharge. Unfortunately, in the experiment 
presented here the $\Delta \mathrm{V} / \Delta \mathrm{t}$ did not change significantly since the peak voltages and their rise times were kept practically constant due to generator limitations.

In general, the cases with detected X-rays presented intense RF levels that the ones without the detection of high energy pulses. The cases that produced breakdown also tend to produce higher peaks of RF. This can be due to the cases with breakdown, the streamer/leaders in the gap produced higher electric field peaks before the breakdown. However, X-rays are also found without breakdown of the gap (not shown here but indicated by March et al. [2012]).

Asymmetry in the RF power production and X-rays is also found between negative and positive discharges. X-rays are measured in negative impulses whereas we have not detected X-rays in positive impulses. Some attention must be paid since the presence of the negative streamers/leaders in the gap can arise from positive impulses depending on the gap geometry. Then, we mention negative impulses to those dominated by a negative streamer/leader and positive impulses to those dominated by positive streamers/leaders in the gap. From figure 3 we can see how positive discharges are efficient producing RF radiation because with lower peak voltages the RF levels are higher than in negative discharges. This is similar to the asymmetry in the breakdown and the critical electric fields for positive and negative streamer propagation (Raizer [1991]).

\section{CONCLUSIONS}

We have demonstrated that X-rays and RF emissions in the microwaves share some common mechanisms. The main mechanism for both radiations would come from interactions of electrons with atoms. Then bremsstrahlung may be the responsible for both 
emissions. The results encourage the study of microwave radio emissions during X-rays produced by natural lightning and during TGF.

\section{ACKNOWLEDGMENTS}

This study was supported by research grants from the Spanish Ministry of Economy and Competitiveness (MINECO) AYA2011-29936-C05-04 and ESP2013- 48032-C5-3-R. We wish to thank LABELEC (Terrassa, Barcelona, Spain) laboratory to allow test execution.

\section{REFERENCES}

Babich L.P. and T. V. Loiko, 2010: Peculiarities of detecting pulses of runaway electrons and X-rays generated by high-voltage nanosecond discharges in open atmosphere, Plasma Physics Reports, 2010, Vol. 36, No. 3, pp. 263-270.

Bekefi, G., and S. C. Brown, 1961: Emission of radio-frequency waves from plasmas, J. Appl. Phys. 32, 25.

Bondiou, A., G. Labaune, J.P. Marque, 1987: Electromagnetic radiation associated with the formation of an electric breakdown in air at atmospheric pressure. J. Appl. Phys. 61 (2), $503-509$.

Brook, M., and N. Kitagawa, 1964: Radiation from lightning discharges in the frequency range 400 to $1000 \mathrm{Mc} / \mathrm{s}$, J. Geophys. Res., 69(12), 2431-2434, doi:10.1029/JZ069i012p02431. 
Cooray, V., and G. Cooray, 2012: Electromagnetic radiation field of an electron avalanche, Atmos. Res., 117, 18-27.

Dwyer, J.R., H.K. Rassoul and Z. Saleh, 2005: X-ray bursts produced by laboratory sparks in air, Geophys. Res. Lett. 32, L20809, doi:10.1029/2005GL023975.

Fishman, G.J., P.N. Bhat, R. Mallozzi, J.M. Horack, T. Koshut, C. Kouveliotou, G.N. Pendleton, C.A. Meegan, R.B. Wilson, W.S. Paciesas, S.J. Goodman, and H.J. Christian, 1994: Discovery of intense gamma-ray flashes of atmospheric origin, Science, 264, 13131316.

Kochkin, P. O., C. V. Nguyen, A. P. J. van Deursen, and U. Ebert, 2012: Experimental study of hard X-rays emitted from metre-scale positive discharges in air, J. Phys. D Appl. Phys., 45, 425202.

Kosarev, E. L., V. G. Zatsepin, and A. V. Mitrofanov, 1970: Ultrahigh frequency radiation from lightnings, J. Geophys. Res., 75(36), 7524-7530, doi:10.1029/JC075i036p07524.

March, V., and J. Montanyà, 2010: Influence of the voltage-time derivative in X-ray emission from laboratory sparks, Geophys. Res. Lett., 37, L19801, doi:10.1029/2010GL044543. 
March, V., and J. Montanyà, 2011: X-rays from laboratory sparks in air: The role of the cathode in the production of runaway electrons, Geophys. Res. Lett., 38, L04803, doi:10.1029/2010GL046540.

March, V., J. Montanyà, D. Romero, G. Sola, O. Van der Velde, 2012: X-rays from laboratory sparks in air: The relationship between runaway electrons and the electric field, Proc. of the International Conference on Lightning Protection (ICLP), doi: 10.1109/ICLP.2012.6344375.

Moore, C. B., K. B. Eack, G. D. Aulich, and W. Rison, 2001: Energetic radiation associated with lightning stepped-leaders, Geophys. Res. Lett. 28, 2141-2144.

Nguyen, C. V., A. P. J. van Deursen, and Ute Ebert, 2008: Multiple X-ray bursts from long discharges in air, J. Phys. D: Appl. Phys., 41, 234012, doi:10.1088/0022$3727 / 41 / 23 / 234012$.

Petersen, D., and W. Beasley, 2013a: Microwave radio emissions of negative cloud-toground lightning flashes, Atmos. Res., doi:10.1016/ j.atmosres.2013.02.006.

Petersen, D. A., and W. H. Beasley, 2013b: High-speed video observations of a natural negative stepped leader and subsequent dart-stepped leader, J. Geophys. Res. Atmos., 118, 12,110-12,119, doi:10.1002/2013JD019910. 
Rahman, M., V. Cooray, N. A. Ahmad, J. Nyberg, V. A. Rakov, and S. Sharma, 2008: Xrays from $80 \mathrm{~cm}$ long sparks in air, Geophys. Res. Lett., 35, L06805, doi:10.1029/2007GL032678.

Rai, J., R. Manoranjan and B. A. P. Tantry, 1972: Bremsstrählung as a Possible Source of UHF Emissions from Lightning, Nature Physical Science 238, 59-60.

Raizer, Y. P., 1991: Gas Discharge Physics, Springer, New York.

Rust, W. D., P. R. Krehbiel, and A. Shalanta, 1979: Measurements of radiation from lightning at $2200 \mathrm{MHz}$, Geophys. Res. Lett., 6(2), 85-88.

Stankevich Y. L., and V.G. Kalinin, 1967: Fast electrons and x-rays at the initial stage of pulsed spark discharge development in air, Sov. Phys. Dokl. 12, 1042-1043.

Takagi, M., T. Takeuti, 1963: Atmospheric radiation from lightning discharges, Proc. Res. Inst. Atmos. Nagoya Univ., 10, 1-11. 

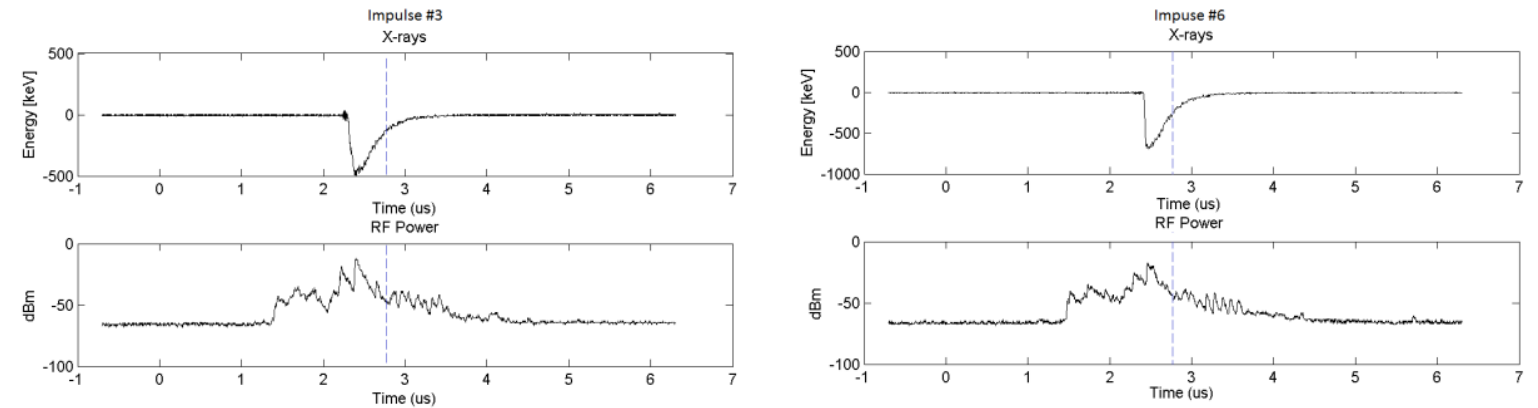

Figure 1. X-rays and RF power at $\sim 2.4 \mathrm{GHz}$ for two negative voltage impulses. Left plot corresponds to a voltage impulse of $-775 \mathrm{kV}$ whereas the right most plot $-786 \mathrm{kV}$. Vertical dashed line indicates the breakdown time. 


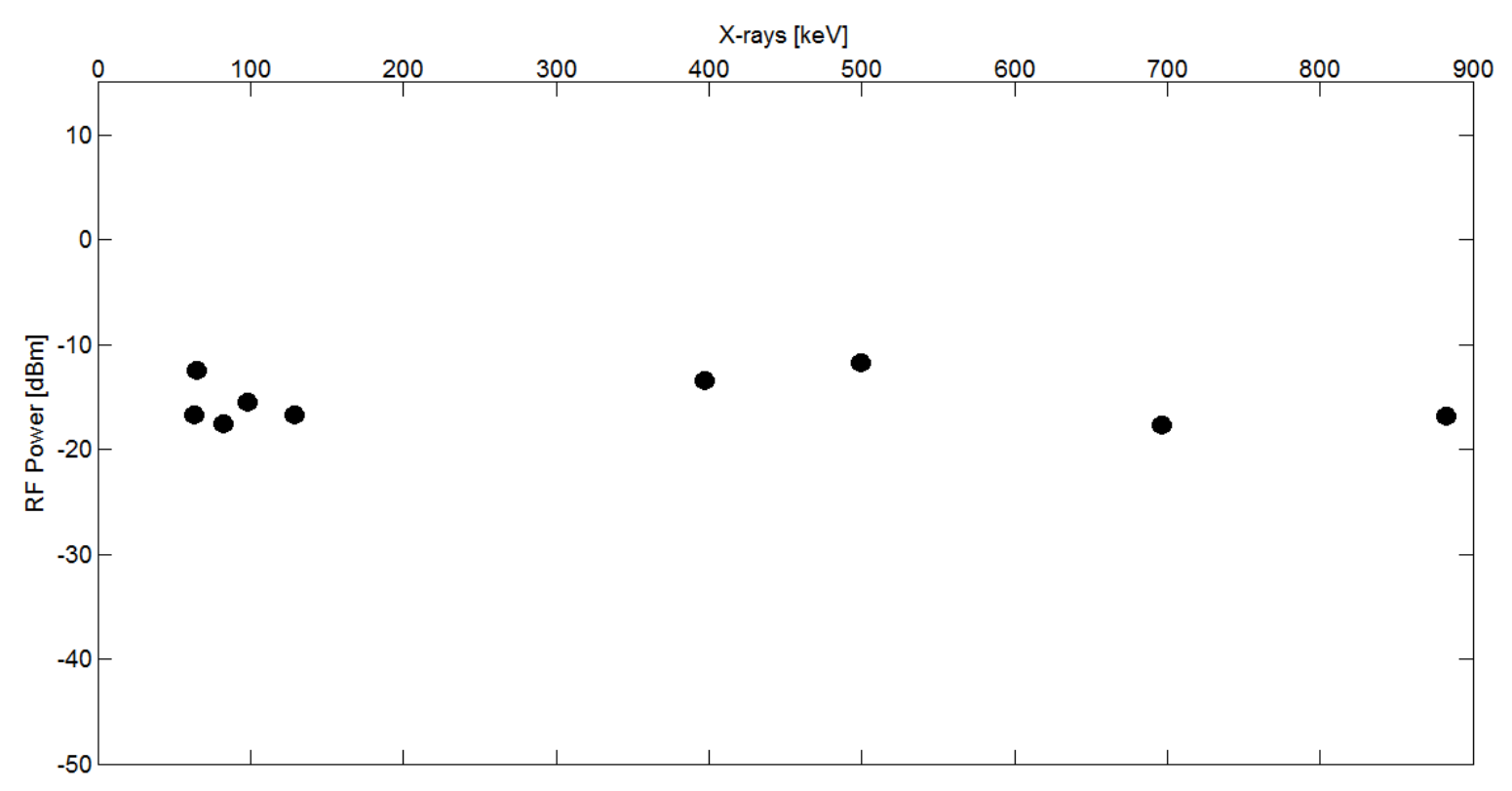

Figure 2. Energy of the detected X-rays versus the RF power.
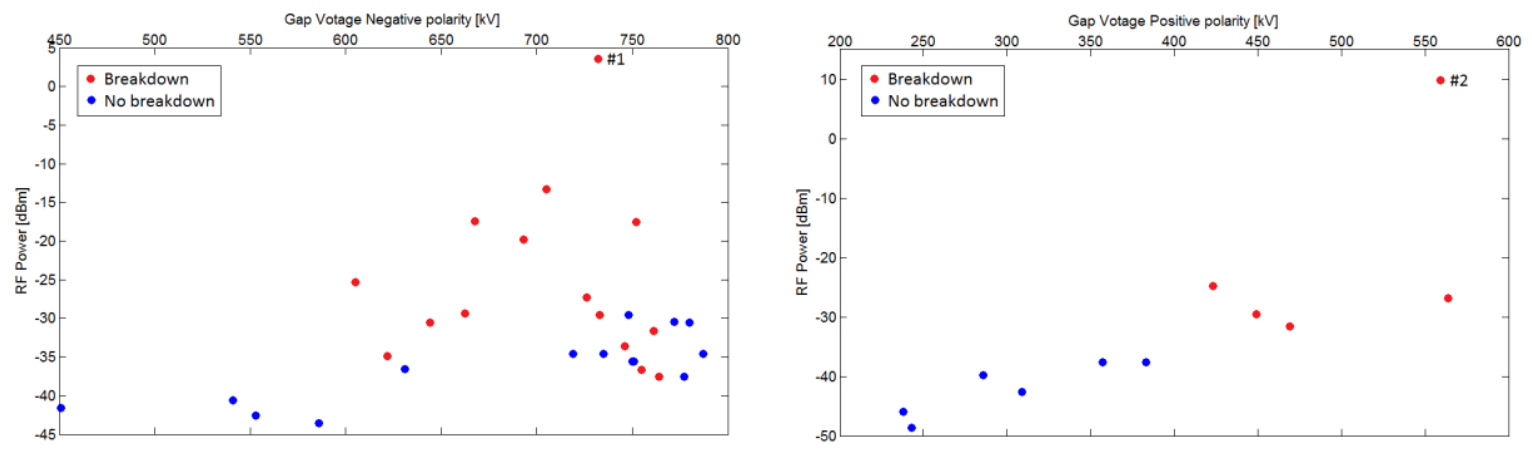

Figure 3. Peak voltage applied to the gap versus RF power at $\sim 2.4 \mathrm{GHz}$ for positive (right) and negative impulses (left). 